\title{
Distributed Ledger Enabled Control of Tyre Induced Particulate Matter in Smart Cities
}

\author{
Panagiota Katsikouli ${ }^{1}$, Pietro Ferraro ${ }^{2 \star}$, Hugo Richardson ${ }^{2}$, Hanson Cheng ${ }^{2}$, \\ Siobhan Anderson ${ }^{2}$, Deepak Mallya ${ }^{2}$, David Timoney ${ }^{3}$, Marc Masen $^{2}$ and \\ Robert Shorten ${ }^{2,3}$ \\ ' Department of Applied Mathematics and Computer Science, Technical University of Denmark, Kongens Lyngby, Denmark, \\ ${ }^{2}$ Dyson School of Design Engineering, Imperial College London, London, United Kingdom, ${ }^{3}$ School of Electric and Electronic \\ Engineering, University College Dublin, Dublin, Ireland
}

\section{OPEN ACCESS}

Edited by:

Emanuele Crisostomi,

University of Pisa, Italy

Reviewed by:

Selim Solmaz,

Virtual Vehicle, Austria Midhat Talibi,

University College London, United Kingdom

*Correspondence: Pietro Ferraro

p.ferraro@imperial.ac.uk

Specialty section:

This article was submitted to Urban Transportation Systems and

Mobility,

a section of the journal Frontiers in Sustainable Cities

Received: 23 June 2020 Accepted: 03 September 2020 Published: 22 October 2020

Citation: Katsikouli P, Ferraro P, Richardson $\mathrm{H}$, Cheng $H$, Anderson S, Mallya D, Timoney D, Masen M and Shorten $R$ (2020) Distributed Ledger Enabled Control of Tyre Induced Particulate

Matter in Smart Cities.

Front. Sustain. Cities 2:575482. doi: 10.3389/frsc.2020.575482
The link between transport related emissions and human health is a major issue for municipalities worldwide and one of the main challenges to address in the context of Smart Cities. Specifically, Particulate Matter (PM) emissions from exhaust and non-exhaust sources are one of the main worrying contributors to air-pollution. In this paper, we challenge the notion that a ban on internal combustion engine vehicles will result in clean and safe air in our cities, since emissions from tyres and other non-exhaust sources are expected to increase in the near future. We support this claim through simple calculations, based on publicly available data from the city of Dublin, and we present a high level solution to this problem, in the form of a control mechanism and ride-sharing scheme to limit the number of vehicles and therefore maintain the amount of transport-related PM to safe levels. Thanks to the use of Distributed Ledger Technology our proposal is entirely distributed, fair and privacy preserving, which makes it ideal for application in the Smart City domain.

Keywords: intelligent transportation systems, control systems, distributed ledger technologies, emission control, non-exhaust emissions

\section{INTRODUCTION}

Smart City research has been the subject of intense interest in the academic community and industry over the past decade (Zambonelli, 2012; Flack and D'Souza, 2014; Miranda et al., 2015; Crisostomi et al., 2017, 2020; Visvizi and Lytras, 2019). This work has given rise to not only new systems of engagement for citizens and their cities, but also to new fundamental research questions in a number of other disciplines. The study of machine learning over "closed loop" data sets is one such example (Epperlein et al., 2019). At a very high level, Smart City Research is about making best use of existing resources in our cities, as we try to manage congestion, pollution, food production, and maintain living standards in the face of ever increasing pressure on natural resources. While managing resources optimally is a classical consideration in many disciplines, new "twists" arise in the context of smart cities that make such problems challenging. Typically, these new "twists" arise as we are often designing systems that are intended to directly interact with humans and influence their behavior. Indeed, in many applications we wish to develop systems to manage the aggregate behavior of large-scale aggregations of populations. Such applications are known to reveal the tension between the good of the individual and societal good, and often what is good for the individual is not always optimal at a societal level, and there are many examples of systems that 
deliver quality of service to the individual at a cost to society. These problems and tensions are often exacerbated by the need to embed "human-centric" notions of privacy, data ownership, trust, fair access to resources, and the need to enforce social compliance, in the populations whose behavior we seek to orchestrate.

Given the above background, our objective in this paper is to consider a problem in cities that, while not presently well-known, is rapidly becoming a pressing concern in cities worldwide. Specifically, we wish to develop algorithms to manage pollution that is associated with tyre abrasion. Much contemporary discussion focusses on mechanisms to abate mobility related greenhouse gases and tailpipe pollutants; see (Crisostomi et al., 2017) for a snapshot of some recent work across several disciplines on this topic. However, as vehicle fleets become electrified, managing non-tailpipe emissions (from tyres and brake disks) is likely to become a very important issue in cities. Our focus in this present paper is to develop algorithms to manage particulate matter arising from tyre abrasion. We use a feedback based access control strategy to manage access to a geographic area in a manner that regulates tyre induced particulate manner to be below a certain value. The use of feedback control is not just due to the fact that we are control engineers. Rather we use feedback to alleviate the need for models of tyre abrasion in a vehicle fleet (which are the subject to great uncertainty), and also to enable the use of distributed ledger technology (DLT) to realize a distributed solution to our problem. Our interest in DLT's is also motivated by some of the softer issues discussed above. More specifically, to address the need for solutions that respect the privacy of individuals, are trust-worthy, and give fair access to the geographic area for all citizens. All of these are important in developing human centric solutions to technical Smart City problems. Finally, the use of DLT's also allow us to manage the community compliance levels building on our initial work in Ferraro et al. (2018).

The importance of managing vehicle emissions is an important topic in cities and link between transport related emissions, and human health, is a major issue for municipalities worldwide. Diesel and other internal combustion engine based motor vehicles are considered to be the major culprit in this regard as they are associated with the generation of a number of harmful emissions. Apart from the link to global warming through the generation of carbon-dioxide, such vehicles are also known to produce other airborne pollutants such as nitrogen oxides, ozone, benzene, carbon monoxide, and particulate matter (PM) of varying size, all of which are considered harmful to human health. In a recent global review (Schraufnagel et al., 2019), it is stated that air pollution, in general, could be damaging every organ and every cell in the human body, showing a potential link between toxic air and skin damages, fertility, asthma and allergies to children and adults.

Amongst others, PM emissions are considered a cause of the aforementioned air pollution (Schraufnagel et al., 2019). PM is a generic term used for a type of pollutants that consist of a complex and varied mix of particles suspended in air. Among all the airborne pollutants PM is particularly worrying due to its ability to enter the bloodstream and reach major organs in the human body. There is rich literature documenting the link between PM and its effects on human health (Laden et al., 2006; Valavanidis et al., 2008; de Miranda et al., 2012; Amato et al., 2014; European Environmental Agency, 2014; Gehring et al., 2015). In particular, the World Health Organization reports that "adverse health effects of PM are due to exposure over both short (hours, days) and long (months, years) terms and include respiratory and cardiovascular morbidity (aggravation of asthma, respiratory symptoms, increase in hospital admissions), as well as mortality from cardiovascular and respiratory diseases and from lung cancer" (WHO, 2013). Smaller PM particles tend to be more harmful to humans compared to larger ones, as they can travel deeper into the respiratory system (WHO, 2013; European Environmental Agency, 2014). Some of the health effects related to PM include oxidative stress, inflammation and early atherosclerosis. Other studies have shown that smaller particles may go into the bloodstream and thus translocate to the liver, the kidneys or the brain (see Grigoratos and Martini, 2014 and references within). Transport related emissions are a significant contributor to airborne PM levels that harm our health. In a recent study (Chen et al., 2017), it is shown that living near major roads (i.e., near emissions from vehicles) is associated with increased risk of dementia. The reduction of air quality and population exposure to harmful pollutants as a result of road passenger transportation is discussed in Hatzopoulou and Miller (2010), in a case study in the Greater Toronto Area. The survey described in Amato et al. (2014) focuses on air pollution originating from non-exhaust emissions such as brake and tyre wear, and highlights the related impact to human health as well as the significance of particulate matter reduction.

Many avenues are being explored worldwide in the fight against urban pollution. Three well-documented examples are the following ones:

(i) Outright bans on polluting vehicles and embracing zero tailpipe emission vehicles in certain city zones (Dey et al., 2018);

(ii) Measuring air quality as a means to better informing citizens of zones of higher pollution (Rai et al., 2017); and

(iii) Developing smart mobility devices that seek to minimize the effect of polluting devices on citizens as they transport goods and individuals in our cities ( $\mathrm{Gu}$ et al., 2017; Herrmann et al., 2018; Sweeney et al., 2018).

Option (i) whereby ultra-low emission zones are created by banning internal combustion engine (ICE) based vehicles in certain areas, in addition to embracing electric vehicles (EV's), has gained much traction worldwide and is being proposed for adoption in cities such as London and Dublin. Apart from the reduced tailpipe pollutants, an additional attraction of the switch from ICE to EV, is that it is beneficial from the perspective of global warming (reduced carbon dioxide), provided that the energy delivered to the EV's can be sourced in a green manner. Thus, reducing our dependency of ICE based vehicles would appear to be very beneficial; not only does the strategy achieve cleaner air but we also potentially tackle climate change through reduced production of carbon dioxide. A major objective of this paper is to challenge the current focus on tailpipe emissions. 
To avoid any misunderstanding, we wholeheartedly endorse a reduced dependency on ICE based vehicles. However, the contemporary narrative is based on tailpipe emissions only, and while it is indeed true that EV's are zero tailpipe emission vehicles, the tailpipe is only one source of PM. Thus replacing one type of vehicle fleet with another type of vehicle fleet, consisting of heavier vehicles, may not result in cities with safe levels of air quality, especially if the non-tailpipe sources of PM are significant.

Among the early solutions to reduce traffic related airpollution has been the application of non-thermal plasma to diesel cars (Chae, 2003). Similar solutions include the application of catalytic filters (Salvat et al., 2000) for reduced exhaust fumes. Such solutions however, fail to address the non-exhaust emissions from diesel and non-diesel vehicles. The potential of road sweeping and washing to reduce non-exhaust related emissions was presented in a study in the Netherlands in 2010 (Keuken et al., 2010). The authors, although they identify non-exhaust emissions as the main source for coarse PM in urban areas, conclude that their approach does not have a significant reduction in non-exhaust emissions. The benefits of ride-sharing to the environment have been discussed in various studies, such as (Concas and Winters, 2007; Chen et al., 2010; Fagnant and Kockelman, 2014; Yu et al., 2017). However, these studies do not take a dedicated interest to non-exhaust emissions, but rather, to fuel consumption reduction. Fuel consumption reduction has been addressed with route suggestion solutions in (Suzuki, 2011; Franceschetti et al., 2013; Coc et al., 2014), for trucks and vehicle fleets. In Franceschetti et al. (2013), the authors present a linear programming solution to the TimeDependent Pollution-Routing Problem. Fleets of vehicles are rerouted depending on traffic, and speeds are recommended based on emissions, driver costs, traffic and peak hour information. As a solution, the authors introduce a departure time and speed optimization algorithm. A similar approach for optimization of fleet size is proposed in Coc et al. (2014). In the same spirit, authors in Mahmod et al. (2013) study a variety of measures, such as traffic control, ban of heavy duty vehicles (HDV) and speed restriction, in order to achieve reduction of traffic related emissions. Traffic control (simulated simply by reducing traffic by $20 \%$ ) and HDV banning have a significant reduction in airpollutants (20-23\%), whereas speed control exhibits increase in PM emissions, due to HDV. Last but not least, use of electric vehicles, as an alternative to diesel and petrol ones, has been suggested for the reduction of traffic related air pollutants. In a feasibility study in Canada and Italy (Longo et al., 2016), the use of electric cars and electric motorcycles shows a reduction in $\mathrm{CO}_{2}$ emissions, however, the study ignores non-exhaust related emissions, which are as relevant to electric vehicles as they are to diesel and petrol ones (Timmers and Achten, 2016). Another study in the city of Dublin (Brady and O'Mahoney, 2011) uses home/work commute and traffic related data to study a number of electric vehicle market penetration scenarios and evaluates the emission decrease under each of them. However, only tailpipe emissions are taken into consideration, again overlooking brake and tyre wear and other non-exhaust emissions. As opposed to the majority of works that address the reduction of road traffic related emissions, we propose a traffic control and ride-sharing scheme, that reduces the amount of cars in the streets, and therefore the tyre-related emissions, as well as other non-exhaust and exhaust emissions.

The contribution of this paper is twofold:

- Use data from Dublin and London to argue that PM levels from tyres alone may be above that which is deemed safe by the World Health Organization (WHO);

- An access control mechanism, based on Distributed Ledger Technology, that both regulates tyre-based PM generation, and provides fair access to a city zone for a set of competing vehicles.

Finally, we want to stress that, while we are by no means the first to argue that tyres are an important source of PM (see in particular the excellent report Kole et al., 2017), we strongly believe it is important that stake-holders be reminded of this message, particularly in the context of the current transitioning from ICE-vehicles toward an electrification of the vehicle fleet.

The remainder of this paper is organized as follows. In section 2, we discuss tyre-wear related PM emissions, supported by numbers from various sources, with a focus on Dublin in Ireland and London in the United Kingdom. The aim of this section is to provide the reader with data and rationale for our Access Control Mechanism, presented in detail in section 3. We simulate our system and present results in Section 4 and conclude the paper in section 5 .

\section{ELEMENTARY CALCULATIONS}

\subsection{Particulate Matter}

PM is the product of brake and tyre wear from vehicles as well as a by-product of the engine combustion process. The most common classification of particulate matter is according to size: $P M_{10}$ for particles with at most $10 \mu \mathrm{m}$ diameter, $P M_{2.5}$ for particles with at most $2.5 \mu \mathrm{m}$ diameter, and ultrafine particles which have a diameter of less than 0.1 micrometers. Smaller PM particles tend to be more harmful compared to larger ones as they are able to get deeper into the respiratory system with ultrafine particles being able to get into the bloodstream and therefore translocate into vital organs such as the liver, the kidneys and the brain.

\subsection{Designated Safe Levels of PM}

According to the $\mathrm{WHO}$, for $P M_{2.5}$, the daily maximum deemed safe level on average is $25 \mu \mathrm{g} / \mathrm{m}^{3}$, whereas the annual maximum permitted level is on average 10. For $P M_{10}$, the maximum permitted levels are on average $50 \mu \mathrm{g} / \mathrm{m}^{3}$ and $20 \mu \mathrm{g} / \mathrm{m}^{3}$ on a daily and annual basis, respectively. In general, non-exhaust emissions (including brake and tyre wear, road surface wear and resuspension of road dust) resulting from road traffic, account for over $90 \%$ of $P M_{10}$ and over $85 \%$ of $P M 2.5$ emissions from traffic (Timmers and Achten, 2016). 


\subsection{Approximate Guess of Airborne PM in Dublin}

To parse these numbers in terms of tyre abrasion for a city with a high volume of cars, we note that in 2014, nearly 28,000 tones of tyre waste was managed in Ireland (Environmental Protection Agency, 2014). Using publicly available data from the Central Statistics Office ${ }^{1}$ in Ireland, in 2018 approximately 540,000 private cars were continuously active in Dublin throughout the year with an average distance traveled of approximately $15,000 \mathrm{~km}$ per vehicle. We assume that approximately $1 / 3$ of the vehicles (i.e., 170,000 vehicles) will change their tyres in a year in Dublin. A tyre is changed when it has reached a tread wear of approximately $2 \mathrm{~mm}$ (or $1.6 \mathrm{~mm}$ as is the legal minimum). This translates into approximately $35,000 \mathrm{~km}$ of traveled distance per vehicle; however, depending on the driving conditions, the traveled distance before a tyre is changed can vary from 10,000 $\mathrm{km}$ (harsh braking and acceleration, constant change of gears) to $80,000 \mathrm{~km}$ (perfect driving conditions and favorable road and weather). Depending on the type of the tyre and the road conditions, a vehicle (i.e., 4 tyres) loses $50-240 \mathrm{mg} / \mathrm{km}$ in mass (Grigoratos et al., 2018), which accounts for $4-6 \mathrm{~kg}$ of tyre mass lost before tyres are changed. By considering $4 \mathrm{~kg}$ of tyre mass lost per vehicle, we estimate that in Dublin, in 2018 , at least $680,000 \mathrm{~kg}$ of tyre mass was wasted, $10 \%$ of which goes airborne (Kole et al., 2017; Grigoratos et al., 2018) as PM. That corresponds to approximately $68,000 \mathrm{~kg}$ of particulate matter in a year, or $186 \mathrm{~kg}$ per day, in the city of Dublin. (Grigoratos et al., 2018) states that approximately 50\% of the $P M_{10}$ emissions (not specifically to air) fall in the $P M_{2.5}$ category. (Fauser et al., 1999) reports that c.90\% of airborne tyre wear particles are smaller than 1 micrometer in diameter (that is, in the $P M_{2.5}$ category). In Grigoratos and Martini (2014), references of previous studies state that $3-7 \%$ of tyre wear particles contribute to airborne $P M_{2.5}$.

\subsection{Disclaimer}

Note that the ones presented above are approximate calculations, in the absence of precise tyre abrasion diffusion models. While a more accurate model, for example based on Panis et al. (2006), would give better estimates of the threat to human health, and is a subject of further work, the previous analysis relies on a macroscopic analysis which, in the compilation of emission inventories of large areas and long time periods, is expected to give reasonably good estimates (Panis et al., 2006). We however realize that the previous calculations are based on a large number of assumptions and a more accurate analysis will be the subject of a future work.

\subsection{Elementary Experiment to Establish Level of Rubber in Road Dust}

Finally, to determine the presence of tyre residuals in road dust in a real urban environment, and the relative proportion of rubber vs. non-rubber particles, six road dust samples were collected from five locations in central London, as depicted in Figure 1. QQueensgate road (high traffic density, location 1 on map);

\footnotetext{
${ }^{1}$ Central Statistics Office. https://www.cso.ie/en/index.html.
}

Imperial College Fallmouth gate (high traffic density, location 2); Cromwell road (high traffic density, location 3); Gloucester road train station (medium traffic density, location 4); and two low traffic locations at Brechin place/Jay Mews (location 5). The locations were chosen to be a mix of low volume, medium volume and high volume traffic density sites and included major thoroughfares and residential streets. London has been chosen to conduct this experiment, because, as Dublin, it represents a capital and the largest city of one of the major countries in Europe. All samples were taken on 17/10/2019. Prior to selecting sites we attempted to correspond the time of collection to be a consistent period post street cleaning. Unfortunately it was not possible to obtain an accurate schedule; cleaning services were found to be run on a continuous loop with no further description of starting point or time, and this is one source of uncertainty in the measurement. Collection consisted of taping a $2 \mathrm{~m} \times 2 \mathrm{~m}$ area, $1 \mathrm{~m}$ from the edge of the road, and collecting dust using a boom and pan over a 1 min period. An "average" urban road dust sample was subsequently created by mixing these various collected samples. In order to measure the amount of tyre dust we employed thermogravimetric analysis (Coats and Redfern, 1963), to measure mass change with temperature increase. The results of this analysis on the aggregated road sample are depicted in Figure 2. Tyre rubber ignites at approximately $700^{\circ} \mathrm{C}$, and at this temperature the thermogravimetric curve shows a drop of $6.6 \%$. This indicates that the mass fraction of rubber (or a similar material) in the collected road dust is approximately $6.6 \%$ (note that this is an approximate measure since in the inert atmosphere, the weight loss is due to polymer and oil volatilizing off Terrill et al., 2010. Therefore, there may be volatiles from other sources than tyre rubber compounds in the dust samples).

Note that while this is a very approximate analysis, and the sampling of the road dust, both limited and primitive, the results are consistent with the view that tyre particulates may be a significant component of road dust.

\section{FEEDBACK-ENABLED ACCESS CONTROL}

The pollution mitigation mechanisms discussed in the previous section, and the move from ICE to EV's that is so popular in many cities globally, is based on the assumption that the principal source of pollution is tailpipe in origin. As we have discussed in the previous section this assumption is at best only partially true and tyres, brakes, as well as road abrasion, may contribute significantly to PM generation. Additionally, EV's are generally heavier than ICE vehicles, potentially affecting tyre wear negatively. This means that the amount of emitted nonexhaust PM might actually even be elevated for EVs. Therefore, one must look for alternative mitigation mechanisms to combat these sources of PM generation. Apart from the obvious move from private to public transport or other modes of transport such a cycling and scooters, one viable solution is to develop an access control mechanism that is based on a feedback control strategy to regulate the safe levels of PM. It is one such strategy that we now develop. 


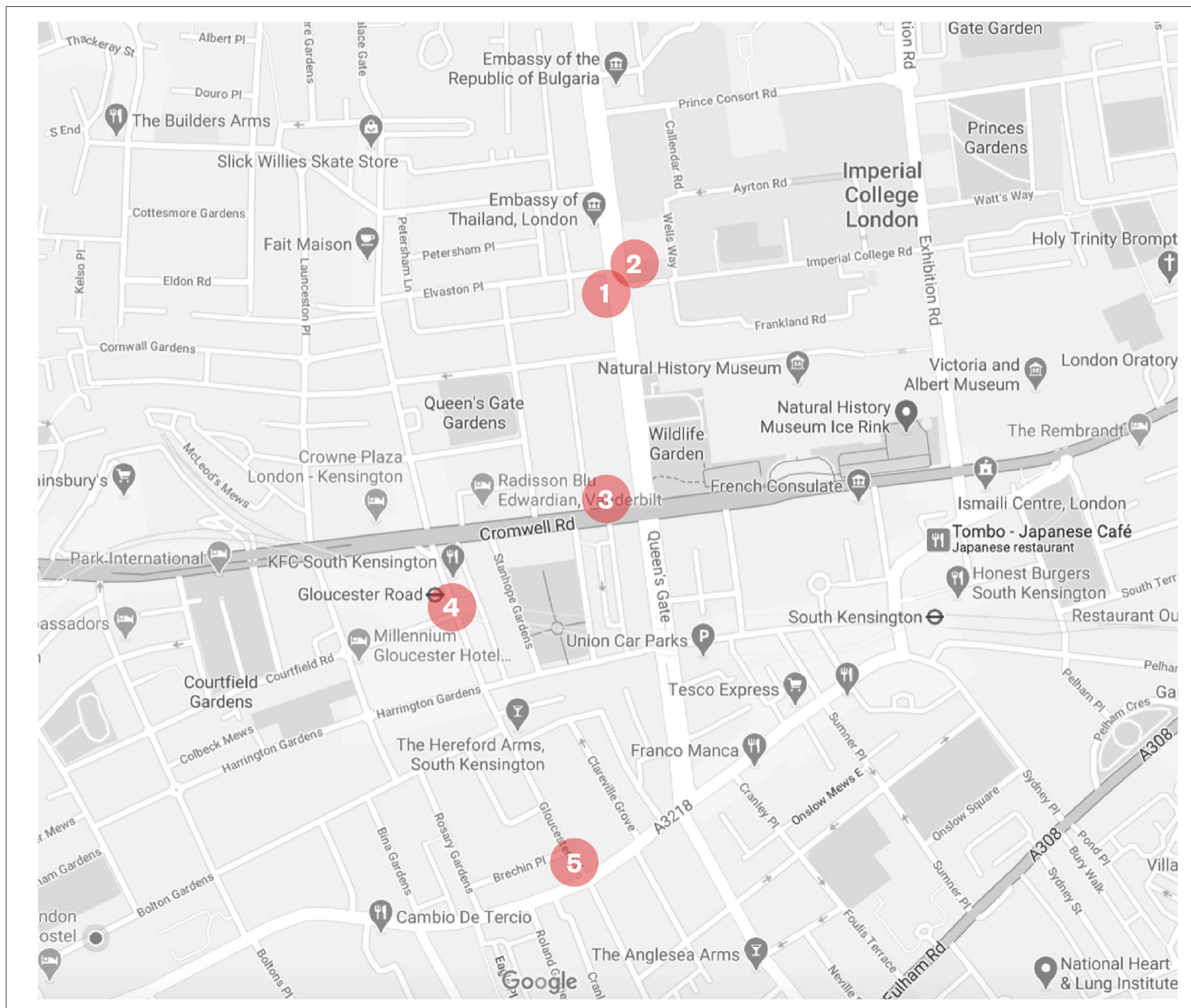

FIGURE 1 | Dust collection locations. Source: https://www.google.it/maps.

Specifically, our objective is to maximize both the number of cars and people entering the city center each day, while maintaining the tyre-generated PM emission levels significantly below the maximum permitted levels. The idea is to orchestrate an access control scheme so that it encourages ride-sharing. The access mechanism works in a simple way: at each day passengers are assigned to cars (drivers) through a matching method. Then, cars who want to have access to the city center are picked randomly using a probabilistic method that ensures fairness and privacy to each user and which is based on occupancy. See Figure 3, for a visual depiction of this scheme.

The rationale behind the choice of a probabilistic method instead of a deterministic one, like a water-filling algorithm, lies in the fact that the latter can be quite inefficient from the single user perspective. In order to use an access control scheme, an agent (driver or passenger) would typically buy a monthly or yearly access pass. This ticket provides them with the opportunity of competing with other users to access the city, either as a driver or as a passenger. Consider now the example of parents, that have to take their children to school outside the city center before traveling into the city center in the morning: even though they paid the same amount for a monthly or yearly parking ticket as everyone else, in a deterministic system they always have a greater chance of missing out the chance of having access to the center of the city, as they arrive later than everyone else. Using a probabilistic system, as the one described in Griggs et al. (2016), we are able to guarantee equality in regards to access for all users over the long-term period of validity of their pass, irrespective of their constraints.

For this Access Control Method, we assume that the controlled region (referred to as $R$ ) can accommodate up to $N$ vehicles per day, decided so that the tyre-related PM emissions 


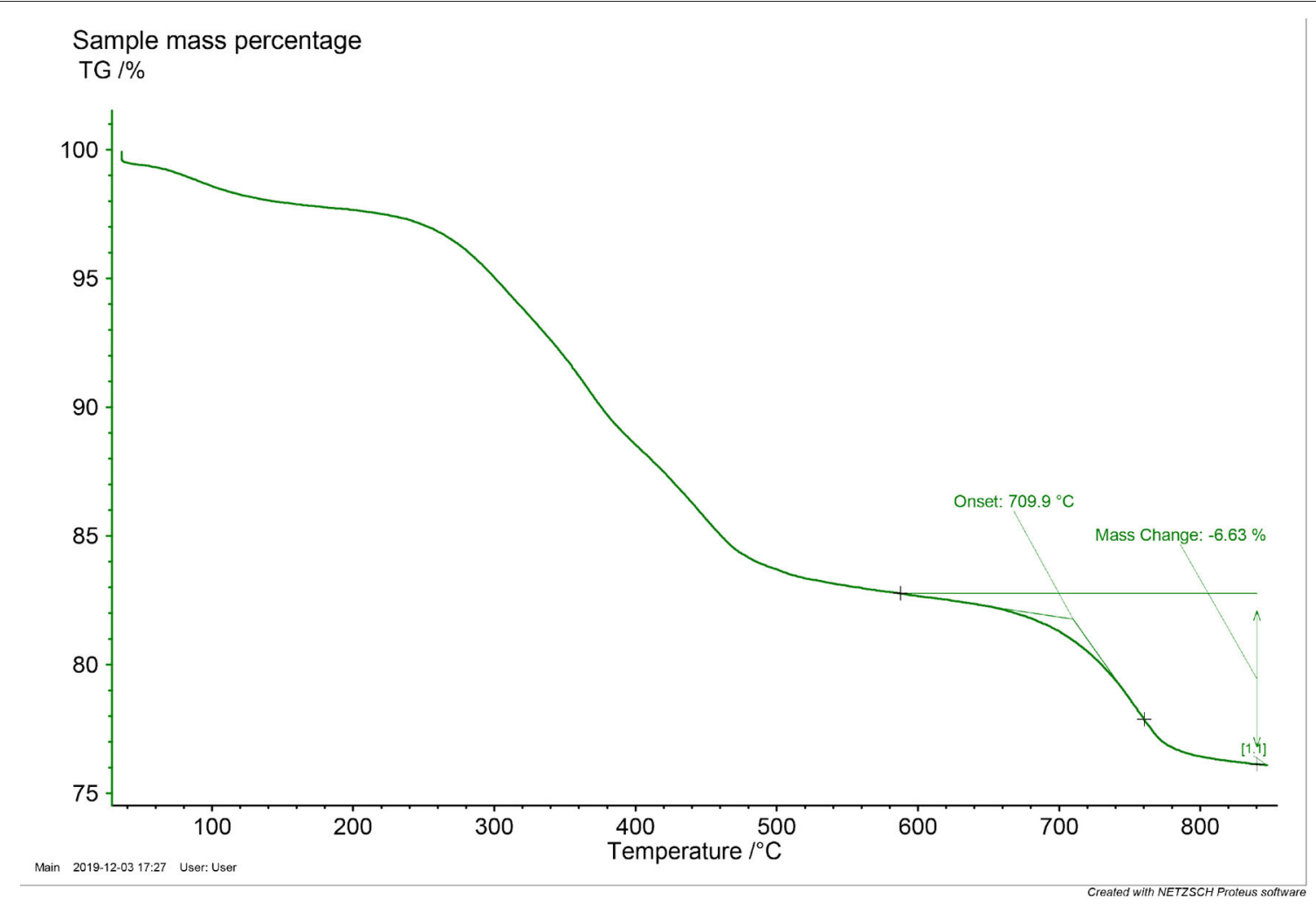

FIGURE 2 | Thermogravimetric analysis of road dust aggregated over all sites.

are kept at low levels, ensuring thus that in general PM emission levels will remain low. There are mainly two challenges to make this method work efficiently.

Q1 Compliance: How does one make sure that users comply with the matchmaking scheme, after access has been granted?

Q2 Fair access: How does one ensure that each driver is granted access to $R$ fairly with respect to other users (for instance, keeping the amount of average access the same among all cars)?

We answer these questions in detail in the following subsections.

Comment: We notice that for the proposed approach to work, the city needs to have a well-connected and well-developed public transport system in order to allow people that are not admitted to the ride-sharing scheme, to travel to the city center. Moreover the control system could be complemented by a premium to encourage the use of public transport, so that only those who truly need to travel with a private vehicle will make use of this scheme.

\subsection{Ride-Sharing Compliance}

In a Ride-Sharing scheme, as the one described above, one of the crucial elements to make the architecture work is to ensure that both drivers and passengers comply with the matchmaking system. While other papers tackle the optimal assignment of passengers and drivers (Alonso-Mora et al., 2017), we are interested in finding methods to maximize the level of compliance in the proposed ride-sharing scheme, and to dynamically regulate vehicle access. The matchmaking mechanism described next is independent of the specific way the passenger/driver assignment is done, and other, alternative, ride sharing mechanisms could also be applied in our overall system. If users are not somehow punished for negative behavior, they might be inclined to cheat the system to maximize their own personal advantage, which in turn might lead to sub-optimal results and to a poor Quality of Service (QoS) overall. As an example, in order to increase the probability of gaining access, a driver might accept as many drivers as possible and then refuse to pick them up; on the other hand, a passenger might choose to not show up, effectively wasting time and resources (the assigned seat). In this context, on the basis of the work done in Ferraro et al. (2018) we propose the use of a digital token as a bond, or digital deposit, to ensure that passengers and drivers comply with their respective social contract (the matchmaking system). The risk of losing a token is then the mechanism that encourages agents to comply with these social contracts. There are multiple practical ways to implement this system: a possible example could be to have each user equipped with a digital wallet and the only way to participate to the matchmaking system is to have enough tokens to use as a bond. Another way could be to link the tokens 

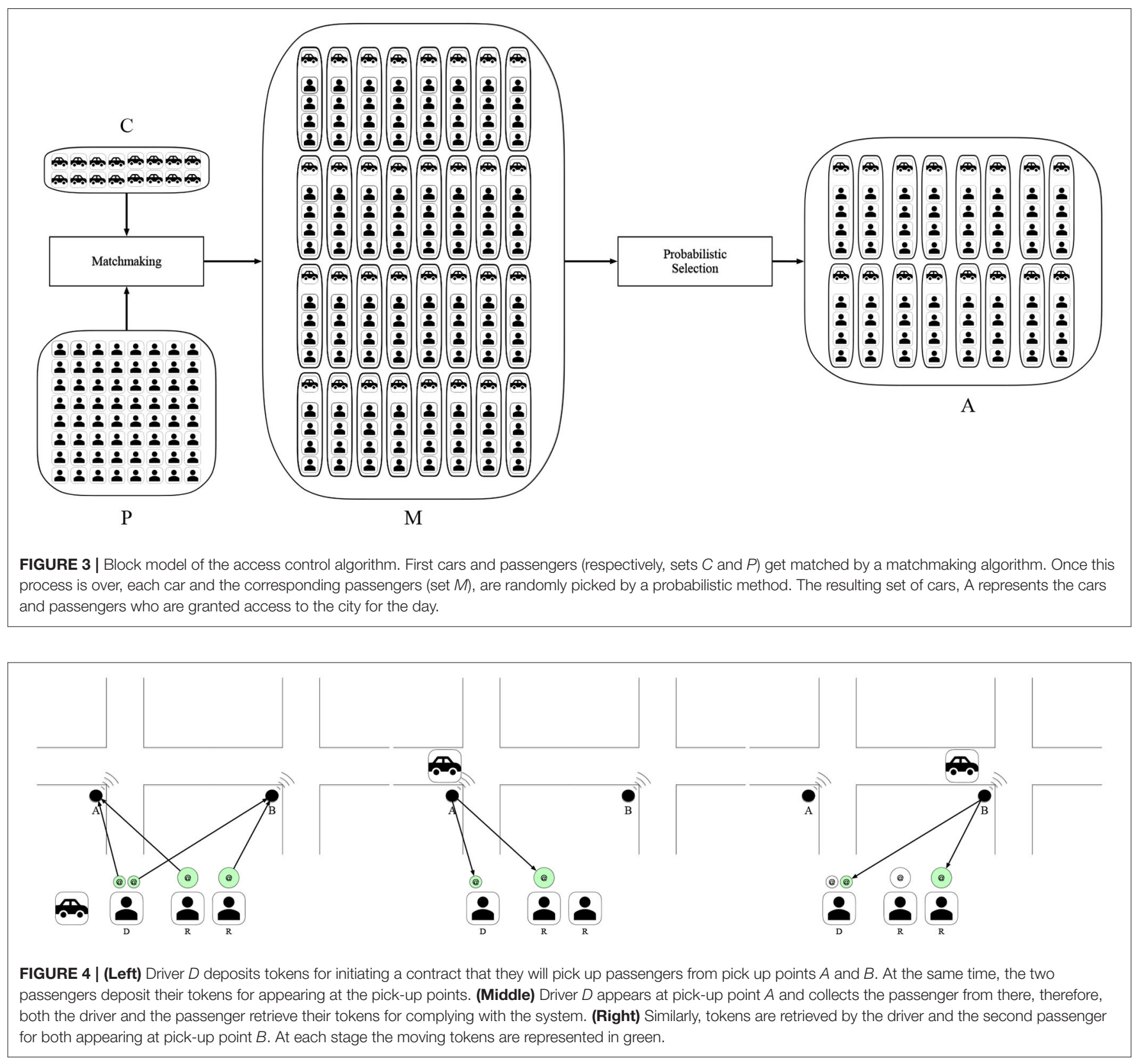

to real money, so that losing a certain amount of them would result in a real economic loss for the agent. Note that the pricing of such tokens is beyond the scope of this paper and is dealt with in Ferraro et al. (2018).

The simple idea is that, whenever a passenger is matched with a driver, they both agree on a specific pick up point and on a time window. Once the passenger gains access to the city center, all the agents involved "deposit" a token to the designed pick up point (notice that this process is repeated between each driver and passenger, therefore a driver will deposit an amount of tokens equal to the number of passengers they are carrying). Then, in order to retrieve their token each agent needs to be physically present at the pick up point, in the designed time window. If unable to do so, the agent will forfeit the possession of the token that can be retrieved by any other passenger/driver present at that time and place. To have a better understanding of this process refer to Figure 4.

In what follows, we propose the use of a permissioned Distributed Ledger Technology (DLT) strategy to implement the proposed access control scheme. The acronym DLT is a term that describes blockchain and a suite of related technologies. From a broad perspective, a DLT is nothing more than a ledger held in multiple places, and a mechanism for agreeing on the contents of the ledger, namely the consensus mechanism. While this technology was first discussed in the white paper from Nakamoto in Nakamoto (2008), the technology has been used primarily 
as an immutable record keeping tool that enables financial transactions based on peer-to-peer trust (Puthal et al., 2018). In order to reach consensus, architectures such as blockchain operate a competitive mechanism enabled via mining (Proof-ofWork), whereas architectures such as the IOTA Tangle (Zheng et al., 2017) based on Directed Acyclic Graph (DAG) structures often operate a cooperative consensus technique. The concept of using tokens to mark specific points where conditions are to be met, perfectly conforms with a DLT-based system. In fact, it is natural to use distributed ledger transactions to update the position of the tokens and to link them to the points of interest and associated data, using transactions (this can be done, for example, using smart sensors linked to digital wallets, as shown in Figure 4). On top of that, a DLT-based system brings a number of advantages as a byproduct of its application to the smart city domain:

- Privacy: In DLTs, transactions are pseudo- anonymous. This is due to the cryptographic nature of the private address ${ }^{2}$, which is less revealing than other forms of digital payments that are uniquely associated with an individual (Esposito et al., 2018). This does not mean that DLTs users' identities are completely anonymous, especially in architectures in which it is possible to follow the trail of transactions among addresses. At the same time though, DLT systems are pseudo-anonymous in the sense that they manage to hide the details of single users and through randomization of the address they can make it difficult for attackers to trace the transactions. Therefore, from a privacy perspective, the use of DLT is desirable in a smart mobility scenario.

- Ownership: Transactions in the DLT can be encrypted, thus allowing every issuer to maintain ownership of their own data. In the aforementioned setting, the only information required to remain public is the current ownership of the tokens, whereas auxiliary information (e.g., user quality of service, statistics on the usage of the system) can be encrypted. This information can later be monetized for the benefit for the data owner.

- Microtransactions: Due to the amount of vehicles in an urban environment, and due to the need of linking the information to real time conditions (such as traffic or pollution levels), there is the demand for a fast and large data throughput.

Furthermore, the DLT system needs to be designed in a way such that whenever a user issues a token as a bond, that same user can retrieve the token if and only if they are present at the pick up zone at the designed time. To do so we make use of the same mechanism and architecture proposed in Overko et al. (2019): namely a Proof of Position (PoP), DAG-based DLT called Spatial Positioning Token (SPToken). Unlike other DLTs, in which each user has complete freedom on how to update the ledger with transactions, the SPToken network has a regulatory policy based on the physical positions of agents. This feature allows for a number of different uses: it can be employed to prevent agents to add transactions that do not possess any relevant data (since transactions can be encrypted) (Overko et al., 2019) or, as in

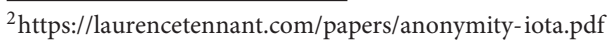

this specific paper, it can be used to make sure that an agent satisfies certain conditions. Therefore, as a validation mechanism, SPToken makes use of PoP to authenticate transactions. In other words, for a transaction to be authenticated, it has to carry proof that the agent was indeed at the pick up point, at the designated time. This is achieved via special nodes called Observers (see Figure 4). Each observer is linked to a physical sensor in a city and it acts as a witness for the transaction. A sensor can be a fixed piece of infrastructure, or a trusted vehicle whose position is verified. As soon as a car is granted access to $R$, each user will deposit their tokens at the designated pick up zone. As soon as an agent reaches in time their pick up point, where one or more of her tokens are available to be picked, a short range connection is established (e.g., via Bluetooth) with the observer (whose job is to authenticate the transaction) and the token is transferred back to the owner's account. Refer again to Figure 4 for a better understanding of this process. This mechanism ensures that users have to be physically present in the interested locations to be able to retrieve their bond. This further authentication step makes SPToken a permissioned DAG-based DLT (similar to permissioned blockchains, Puthal et al., 2018), i.e., a distributed ledger where a certain amount of trusted nodes (the observers, in this case) is responsible to maintain the consistency of the ledger (as opposed to a public one, where security is handled by a cooperative consensus mechanism, Nakamoto, 2008).

Comment: Before continuing, we want to stress that very often in the context of smart cities, algorithms assume full compliance with policies that are designed to optimize the resource allocation. To assume that a human agent would not break rules, especially if an individual profit can be made, is a very strong hypothesis that if relaxed might lead the whole system to fail and to produce less than optimal results. Therefore, it is the authors' opinion that the use of a compliance system is of paramount importance in the setting described so far, if efficiency is to be achieved. The issue of compliance is often overlooked.

\subsection{Mechanism Description}

We consider now the problem of allocating a certain amount of resources (i.e., permitted number of cars) among a set of agents (i.e., drivers and passengers using the scheme). The proposed method is inspired by the algorithm presented in Griggs et al. (2016), appropriately adjusted to the requirements of our ridesharing scheme.

We consider the following scenario. There is a population of size $n$ of citizens participating in the scheme, who request to commute to $R$ on a daily basis. The controlled region can accommodate up to $N$ vehicles per day. We assume $n>N$ and the population could be either passengers or drivers. We assume that there is a fleet of $N^{\prime}>N$ electric vehicles in the scheme that are requested by the population for access in $R$, with $n>N^{\prime}$. Without loss of generality and to facilitate presentation of our mechanism, the entities driver and car are considered equivalent and the corresponding terms are thus used interchangeably. As already mentioned in a previous section, our method is organized in two phases: matchmaking and probabilistic access. During matchmaking, we match passengers with drivers and group them into cars. The matching can happen in a number of ways, 
depending on the specific requirements of those who apply the system. For example, passengers could be matched with drivers based on proximity of their departing/arriving area, or based on a preference priority ranking that drivers/passengers maintain for each other. In our simulations we take a simple approach and match passengers randomly with drivers (and subsequently with cars), as long as there are available seats in the vehicles, taking into consideration the frequency at which a particular passenger has been assigned a seat in the past. That is, if a passenger has been assigned a seat less than $50 \%$ of the time, then they are given priority to take a seat in a car, otherwise, they are not given priority. After the matchmaking is complete, each car is assigned an access probability based on its occupancy records. All cars with high enough probability, are permitted access to the city center. We present the technical details of this procedure, next.

In our system, we will use $k$ to denote number of days (i.e., $k=0,1,2,3, \ldots)$. For ease of interpretation we assume that access is granted on a daily basis to each user, but the algorithm is not affected by this assumption. Then, $X_{i}(k)$ is the state variable associated with each driver; it takes the value 1 if the $i$ th driver is given access to $R$ on the $k$ th day and zero otherwise. Thus, $\bar{X}_{i}(k)$ is the average access for the $i$ th driver up to the $k$ th day, defined as

$$
\bar{X}_{i}(k)=\frac{1}{k+1} \sum_{j=0}^{k} X_{i}(j)
$$

In the above context, let $z_{i} \in[0,1]$ represent the frequency of accessing the city for a car $i$, and $f_{i}:[0,1] \rightarrow \mathbb{R}$ be a convex cost function associated with it, representing the car's priority during the second phase of our mechanism. In this context the shape of this function can take into account a variety of factors: the amount of money paid for the pass (e.g., premium and standard account), the amount of public transportation available in the area where this user lives or the type of vehicle driven. Following (Griggs et al., 2016), we are interested in solving the following shared-resource optimization problem,

$$
\begin{array}{cc}
\underset{z_{1}, \ldots, z_{N^{\prime}} \in \mathbb{R}}{\operatorname{minimize}} & \sum_{i=1}^{N^{\prime}} f_{i}\left(z_{i}\right) \\
\text { subject to } & \sum_{i=1}^{N^{\prime}} z_{i}=N, \\
& z_{i} \geq 0, \quad i=1, \ldots, N^{\prime} .
\end{array}
$$

Our aim is then to control the value of the variable $X_{i}(k)$ (i.e., the access to $R$, at each time step) in such a way that the average access of user $i, \bar{X}_{i}(k)$, converges to the optimal value $z_{i}^{*}$, subject to $\sum_{i=1}^{n} X_{i} \approx N$ (notice that we are not requesting the algorithm to exactly match the required amount of cars, at each time step but we are instead interested in obtaining $\left.\lim _{k \rightarrow \infty} \sum_{i=1}^{n} \bar{X}_{i}(k)=N\right)$. In order to do so, the probability that at each time step car $i$ gains access to the city center (i.e., $X_{i}(k)=1$ ) is ruled by the following equations:

$$
p_{i}(k) \triangleq \mathbb{P}\left(X_{i}(k)=1\right)=\Gamma(k) \frac{\bar{X}_{i}(k)}{f_{i}^{\prime}\left(\bar{X}_{i}(k)\right)} \frac{n_{i}(k)}{c_{i}}
$$

$$
\Gamma(k+1)=\Gamma(k)+\alpha\left(N-\sum_{i=1}^{N^{\prime}} X_{i}(k)\right),
$$

where $n_{i}(k)$ is the number of passengers carried in car $i$ at time $k, c_{i}$ is the car's maximum capacity and $\Gamma(k)$ is a global scaling variable, dependent on the parameter $\alpha>0$, whose dynamics ensures $p_{i}(k) \in[0,1], \forall i, k$. Notice that, Equation (2) differs from the one proposed in Griggs et al. (2016) by the factor $n_{i}(k) / c_{i}$ : since we are interested in maximizing the amount of people getting into $R$ (while maintaining the amount of users having access close to $N$ ), this factor ensures that a fully filled car will have higher probability to be granted access than an empty one. A discussion on the convergence of this algorithm is beyond the scope of this paper and the interested reader can refer to Griggs et al. (2016) for further details.

Comment: Finally, we note that using $N$ as a proxy for tyre emissions may be problematic as it does not take into account the trip distribution associated with $N$. Under the assumption that this distribution is pseudo-stationary over some interval (for example, over consecutive Mondays) then one may use a outer feedback to determine the $N$ corresponding to a certain average emissions level over some interval of time:

$$
N(p+1)=N(p)+c\left(E_{\mathrm{PM}}^{*}-\bar{E}_{\mathrm{PM}}(p)\right)
$$

with $c>0$ being a design parameters, $\bar{E}_{\mathrm{PM}}(p)$ being the filtered (averaged) measured amount of PM emissions at time $p$, averaged over some time period, and $E_{\mathrm{PM}}^{*}$ being the desired level of emissions (which could be, for instance, the average density of $P M_{2.5}$ detected in $\mathrm{R}$ ). Here, as we have mentioned, $p$, and $p+1$, may denote an adjustment from day to another day where the distribution of trips is assumed to be pseudostationary or varying slowly. Note that a measurement of tyre induce emissions can be made using local estimates of traveled distances on individual vehicles, and aggregated at the cloud, or perhaps using devices such as the one proposed $\mathrm{in}^{3}$ (these are claimed to be $60 \%$ efficient leaving $40 \%$ of tyre dust uncollected). Again, individual measurements can be aggregated in the cloud to provide an estimate of the tyre dust generated.

\section{SIMULATIONS AND RESULTS}

We now present empirical results to illustrate the efficacy of the techniques presented in the previous section. In what follows we based our simulations on the recent report (Canal Cordon Report, 2017). All presented simulations are performed on Python, and we make the following assumptions:

- Cars behave in a binary way: they either contribute to the pollution levels or they do not contribute at all (according to Equation 2);

- Each car's emission are computed according to the average values provided in section 2 .

While these can be considered rather simplistic assumptions that do not take into consideration aspects such as the road

\footnotetext{
${ }^{3}$ The Tyre Collective. Available online at: https://www.thetyrecollective.com/.
} 


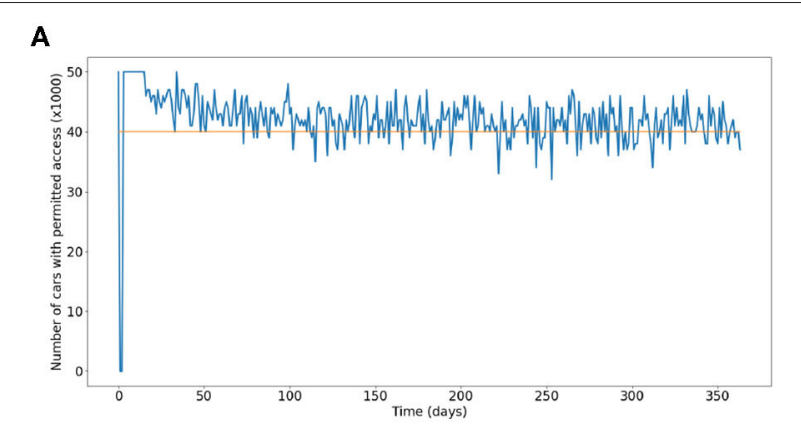

B

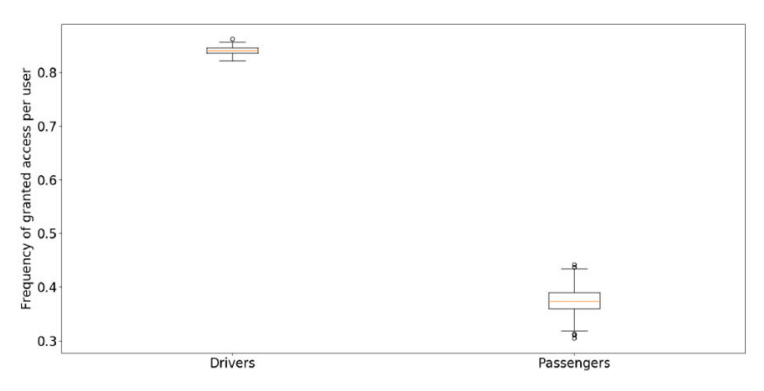

FIGURE 5 | (A) Number of cars with granted access in the length of a year. (B) Frequency of granted access per user in the scheme.

network, road conditions, or the individual behavior of vehicles, for analysis over very large areas (such as the city of Dublin) and over large time scales, the microscopic behavior of single agents is averaged out by the aggregate nature of the scenario (Panis et al., 2006). Therefore, it is the authors' opinion that, while the following results are not suited to provide any quantitative directions for policymakers, they nevertheless provide a qualitatively accurate estimate of the performance of our control system over very large areas. Finally, we point out that this approach represents a high level strategy and is well-suited to be complemented with other measures that would provide a finer resolution to contain PM levels in the context of a Smart City (access to specific areas of the region $\mathrm{R}$, for example).

We assume a city of the size of Dublin in Ireland, with population 1,100,000 approximately, of which 50,000 are considered drivers and about 400,000 are daily commuting passengers (in the report Canal Cordon Report, 2017 it is stated that between 7 and 10 a.m., about 210,000 commuters enter the city center. We make the assumption that in the length of the day that number can potentially double and therefore consider a population of 400, 000 commuters). Consequently, we have a fleet of 50,000 EV's, out of which only 40,000 are permitted in the city center $R$ on a daily basis(in the report Canal Cordon Report, 2017 it is stated that between 7 and 10 a.m., about 50,000 cars entered the city center. Therefore, we limit the number of drivers to that number and the number of permitted cars to slightly less than this figure). All users that are not granted access to the city on a $E V$, are redirected to use public transportation. In our simulations, we set $\Gamma[0]=1$, that is, the value that the parameter $\Gamma$ takes the first day of the scheme's operation, and $\alpha=0.0001$. We also consider an application period of 360 days, that is slightly less than a year long. For convenience, on the first day of the operation, we consider that all drivers are permitted access.

The simulation results are presented in Figures 5A,B. Figure $5 \mathbf{A}$ shows the number of cars that are granted access every day. Although at the beginning the number of cars in area $R$ are above the maximum permitted number $(40,000)$, due to the effect of the access control mechanism this value is quickly reduced, stabilizing around the maximum level, on average, for the rest of the application period. Notice that if the maximum number of drivers in the city center was a hard constraint, it would be sufficient to reduce $N$ to take into account the fluctuations around this value. In Figure 5B, we show the frequency of being granted access, per user, on average over a period of 1 year. The small variance indicates that each user is granted fair access to the system. Regarding the commuting (shared) passengers, every passengers is granted access more than $1 / 3$ of the time.

Figures 6A-E depict the number of cars with access, when the number of maximum permitted drivers changes and all other parameters in the system remain the same. The plots depict the steady state values. We observe that in all cases, the number of cars with granted access converges to the maximum value, on average. In terms of fair access, we show in Figure 6F boxplots of the frequency at which each driver is granted access to the scheme, in the length of a year, with regards to the maximum number of cars permitted in $R$. As expected, the frequency increases as the available amount of resources increases. We highlight that in all cases, the variance is very small, meaning that all drivers in the scheme are ensured fair access (i.e., all drivers are able, on average, to access the city center the same number of times).

Finally to prove the efficacy of our approach in a more dynamic setting, we consider a scenario where we allow the number of maximum permitted cars to vary during the year. There are many reasons that make this a realistic scenario: the city municipality might wish to increase the number of permitted vehicles for the holiday seasons, or reduce it during heat waves, for example. We simulate this setting by changing the number of allowed cars during the year and we present the results in Figure 7. Here, for the first 2 months of the operating period (i.e., 60 days) we give access to $N=50 k$ vehicles. For the next 40 days $N$ increases linearly and for in the interval (of days) $[100,180]$ it is set at $N=80 k$ vehicles. After that, the number of permitted cars decreases linearly again until it is set to the initial value, $N=50 k$, for the rest of the operating period. As we observe in the plot, our system reliably controls the access of vehicles, maintaining the number of permitted cars on average at a stable level around the set of maximum values.

Regarding pollution levels caused by the $P M_{2.5}$ pollutant coming just from the tyre wear of vehicles, we present in Figure 8 the amount of particulate matter, depending on two variables: 

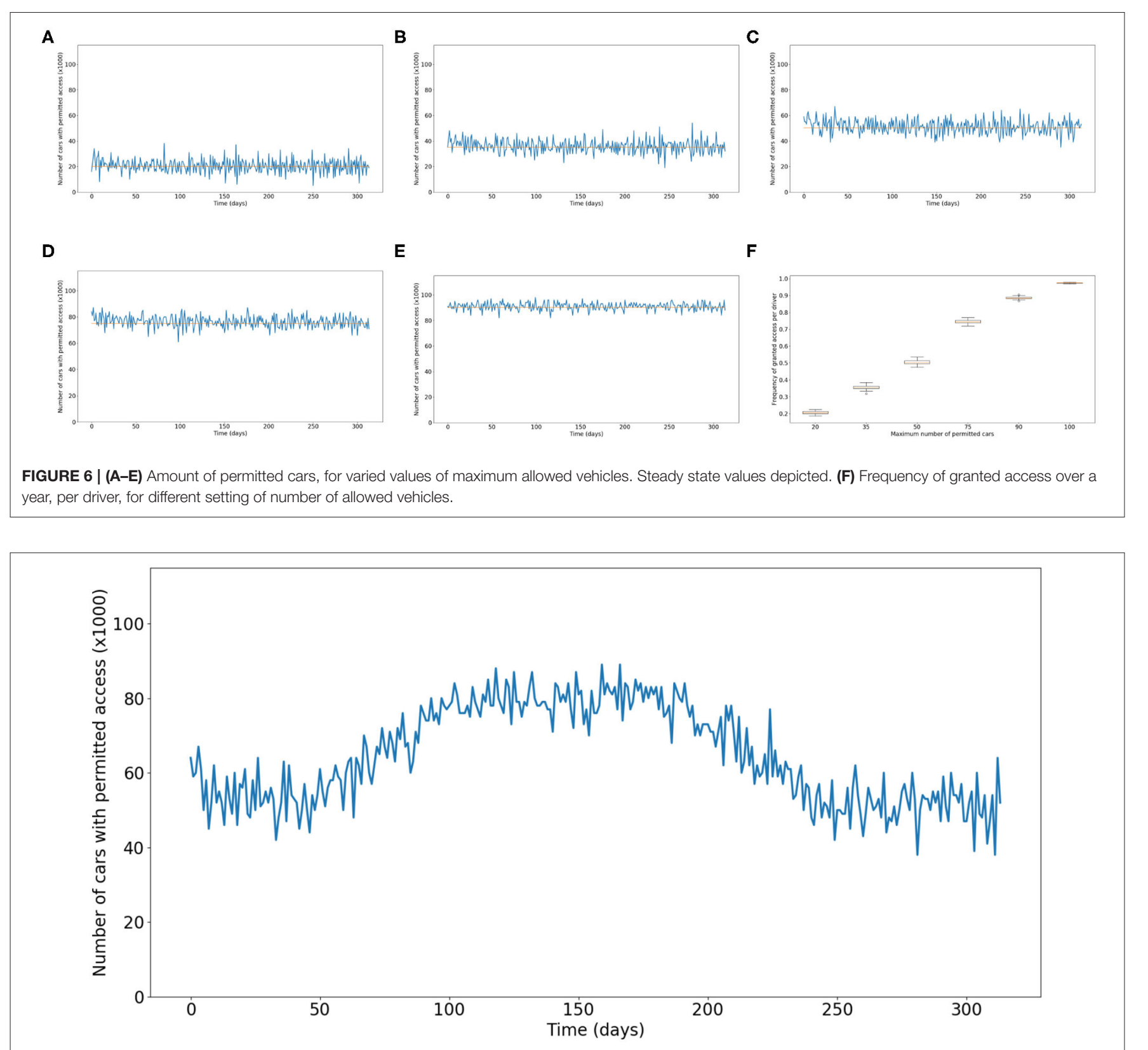

FIGURE 7 | Amount of permitted cars, while changing the value of maximum allowed vehicles gradually from $N=50 \mathrm{~K}$ to $N=80 \mathrm{k}$ and vice versa.

number of cars permitted in a city (Figure $\mathbf{8 A}$ ) and the volume of road network in a city (Figure 8B). With regards to the volume of a city's road network, we wish to estimate, very approximately, the air-space in which the airborne PM is dispersed. For this, we assume that the total mass of PM generated per hour becomes uniformly dispersed throughout a volumetric space which is determined by the street length, an average street width of 10 $\mathrm{m}$ and effectively enclosed by an average building height of $4 \mathrm{~m}$. Furthermore, we assume rather simplistically that the air in this volume is continuously replenished with an equivalent volume of fresh clean air at a rate of one air change per hour, in such a way as to maintain a pollution level which remains effectively constant with time. For the length of the road network, we can compute the total length of the streets in a predefined area in a city. Note that even though $4 \mathrm{~m}$ is a somewhat arbitrary number for these simulations, the basic points remain valid irrespective of this assumption; that the amount of tyre generated PM can be regulated using our access control method. To this end, and based on the above assumptions, Figure $\mathbf{8}$ depicts the amount of $\mathrm{PM}$ per $\mathrm{m}^{3}$ as a function of the number of vehicles operating in a city, per possible volume of space (computed as described above). In these figures, we depict in green the levels deemed safe for human health (i.e., the ones below the maximum permitted levels) and in red the ones exceeding the annual permitted levels. 


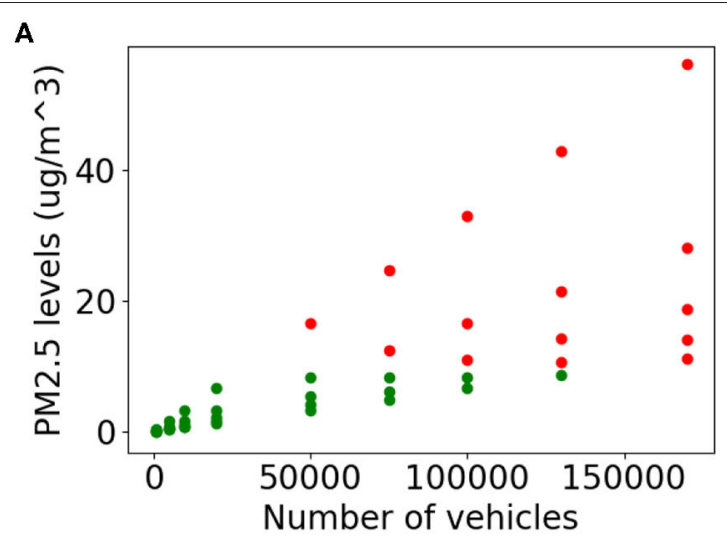

B

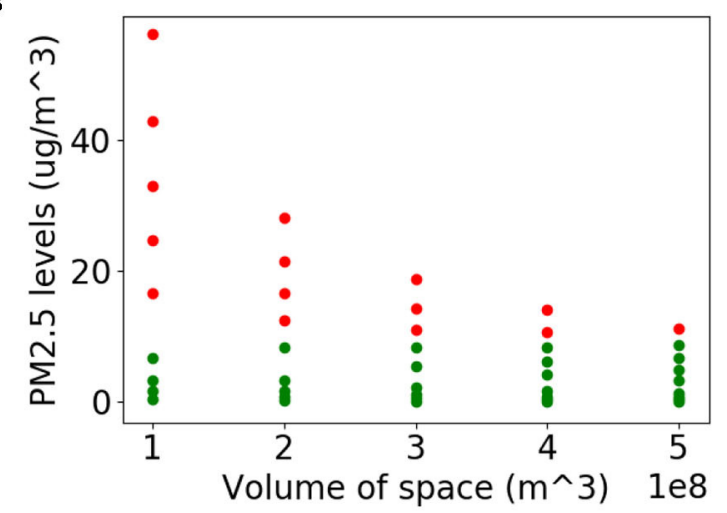

FIGURE 8 | Levels of tyre-wear related $P M_{2.5}$ emissions per number of vehicles operating (A) and volume of space where the matter is dispersed (B).

These plots suggest that, with the present situation in Dublin city (that is, 500,000 cars out of which 170,000 change tyres every year, and a space volume of approximately $450,000,000 \mathrm{~m}^{3}$ ), the levels of tyre-wear related $P M_{2.5}$ emissions are very high. However, applying an access control scheme that restricts the number of vehicles to at most 100,000 vehicles per day, can maintain the PM levels at acceptable levels even in small size cities with relatively small volume of space.

\section{CONCLUSIONS}

This paper proposes the use of an access control mechanism, to reduce the pollution levels and the health hazard caused by nonexhaust emissions, in smart cities. The contributions of this paper are divided into two sections. In the first one, a detailed data analysis shows suggests that a simple ban on ICE vehicles does not address the problem of non-exhaust emissions (PM from tyres, in particular). Although there have been previous studies that present such numbers for other cities, we emphasize the point that in Dublin, the PM levels from tyres alone might be above the levels that are deemed safe by WHO. This provides us with the rationale to introduce, in the second part, an access control and ride-sharing scheme to limit the amount of cars in cities and therefore maintain the amount of airborne PM within safe levels for our health. This system is designed in such a way to encourage users to comply with the matchmaking scheme and to guarantee fair access to each car. Very often in the context of smart cities, algorithms assume full compliance with policies that are designed to optimize the resource allocation. To assume that a human agent would not break rules, especially if an individual profit can be made, is a very strong hypothesis that if relaxed might lead the whole system to fail and to produce less than

\section{REFERENCES}

Alonso-Mora, J., Samaranayake, S., Wallar, A., Frazzoli, E., and Rus, D. (2017). Ondemand high-capacity ride-sharing via dynamic trip-vehicle assignment. Proc. Natl. Acad. Sci. U.S.A. 114, 462-467. doi: 10.1073/pnas.1611675114 optimal results. Therefore, it is the authors' opinion that the use of a compliance system is of paramount importance in the setting described so far, if efficiency is to be achieved. Finally, to validate the proposed algorithm, we make use of extensive simulations to show that each user receives fair access to the city center and that the PM emissions are kept within safe boundaries. As for future lines of research we will further extend the present work by using more complex models for tyre abrasion and airborne diffusion to obtain more accurate estimates for non exhaust emissions and we will extend the access control strategy to take into consideration complex networks of roads and the possible adverse consequences of such a policy.

\section{DATA AVAILABILITY STATEMENT}

Publicly available datasets were analyzed in this study. This data can be found here: https://www.cso.ie/en/index.html.

\section{AUTHOR CONTRIBUTIONS}

All authors contributed equally to the writing of this manuscript.

\section{FUNDING}

This work was partially supported by SFI grant 16/IA/4610 and partially funded by the IOTA Foundation.

\section{ACKNOWLEDGMENTS}

The content of this manuscript has been released as a pre-print at https://arxiv.org/ (Katsikouli et al., 2019). 
Transport. Res. D Transport Environ. 16, 188-193. doi: 10.1016/j.trd.2010. 09.006

Canal Cordon Report (2017). Report on Trends in Mode Share of Vehicles and People Crossing the Canal Cordon 2006-2017.

Chae, J. O. (2003). Non-thermal plasma for diesel exhaust treatment. J. Electrostat. 57, 251-262. doi: 10.1016/S0304-3886(02)00165-1

Chen, H., Kwong, J.C., Copes, R., Tu, K., Villeneuve, P.J., Van Donkelaar, A., et al. (2017). Living near major roads and the incidence of dementia, Parkinson's disease, and multiple sclerosis: a population-based cohort study. Lancet 389, 718-726. doi: 10.1016/S0140-6736(16)32399-6

Chen, P. Y., Liu, J. W., and Chen, W. T. (2010). "A fuel-saving and pollutionreducing dynamic taxi-sharing protocol in VANETs," in IEEE 72 nd Vehicular Technology Conference, 1-5.

Coats, A. W., and Redfern, J. P. (1963). Thermogravimetric analysis. A review. Analyst 88, 906-924.

Coc, C., Bekta, T., Jabali, O., and Laporte, G. (2014). The fleet size and mix pollution-routing problem. Transport. Res. B Methodol. 70, 239-254. doi: 10.1016/j.trb.2014.09.008

Concas, S., and Winters, P. L. (2007). Impact of carpooling on tripchaining behavior and emission reductions. Transport. Res. Rec. 2010, 83-91. doi: 10.3141/2010-10

Crisostomi, E., Ghaddar, B., Hausler, F., Naoum-Sawaya, J., Russo, G., and Shorten. R. (eds.). (2020). Analytics for the Sharing Economy. Springer.

Crisostomi, E., Shorten, R., Studli, S., and Wirth, F. (2017). Electric and Plug-in Hybrid Vehicle Networks. CRC-Press.

de Miranda, R. M., de Fatima Andrade, M., Fornaro, A., Astolfo, R., de Andre, P. A. and Saldiva, P. (2012). Urban air pollution: a representative survey of $P M_{2.5}$ mass concentrations in six Brazilian cities. Air Qual. Atmos. Health 5, 63-77. doi: 10.1007/s11869-010-0124-1

Dey, S., Caulfield, B., and Ghosh, B. (2018). Potential health and economic benefits of banning diesel traffic in Dublin, Ireland. J. Transport Health 10, 156-166. doi: $10.1016 /$ j.jth.2018.04.006

Environmental Protection Agency (2014). Waste Tyre Statistics for Ireland.

Epperlein, J., Shorten, R., Zhuk, S. (2019). Recovering Markov models from closedloop data. Automatica 103, 126-135. doi: 10.1016/j.automatica.2019.01.022

Esposito, C., De Santis, A., Tortora, G., Chang, H., and Choo, K. K. R. (2018). Blockchain: a panacea for healthcare cloud-based data security and privacy? IEEE Cloud Comput. 5, 31-37. doi: 10.1109/MCC.2018.011791712

European Environmental Agency. (2014). Air Quality in Europe 2014 Report. Available online at: http://www.eea.europa.eu/publications/air-quality-ineurope- 2014

Fagnant, D. J., and Kockelman, K. M. (2014). The travel and environmental implications of shared autonomous vehicles, using agent-based model scenarios. Transport. Res. C Emerg. Technol. 40, 1-13. doi: $10.1016 /$ j.trc.2013.12.001

Fauser, P., Tjell, J. C., and Bjerg, P. L. (1999). Particulate air pollution, with emphasis on traffic generated aerosols. (Ph.D. thesis).

Ferraro, P., King, C., and Shorten, R. (2018). Distributed ledger technology for smart cities, the sharing economy, and social compliance. IEEE Access 6, 62728-62746. doi: 10.1109/ACCESS.2018.2876766

Flack, J. and D'Souza, R. (2014). The digital age and the future of social network science and engineering. Proc. IEEE 102, 1873-1877. doi: 10.1109/JPROC.2014.2368790

Franceschetti, A., Honhon, D., Van Woensel, T., Bekta, T., and Laporte, G. (2013). The time-dependent pollution-routing problem. Transport. Res. B Methodol. 56, 265-293. doi: 10.1016/j.trb.2013.08.008

Gehring, U., Beelen, R., Eeftens, M., Hoek, G., de Hoogh, K., de Jongste, J., et al. (2015). Particulate matter composition and respiratory health: the PIAMA Birth Cohort Study. Epidemiology 26, 300-309. doi: 10.1097/EDE.0000000000000264

Griggs, G. Yu, J., Wirth, F., Hausler, F., and Shorten, R. (2016). On the design of campus parking systems with QoS guarantees. IEEE Trans. Intell. Transport. Syst. 17, 1428-1437. doi: 10.1109/TITS.2015.2503598

Grigoratos, T., Gustafsson, M., Eriksson, O., and Martini, G. (2018). Experimental investigation of tread wear and particle emission from tyres with different treadwear marking. Atmos. Environ. 182, 200-212. doi: 10.1016/j.atmosenv.2018.03.049
Grigoratos, T., and Martini, G. (2014). Non-Exhaust Traffic Related Emissions. Brake and Tyre Wear PM.

Gu, Y., Liu, M., Naoum-Sawaya, J., Crisostomi, E., Russo, G., and Shorten, R. (2017). Pedestrian-aware engine management strategies for plug-in hybrid electric vehicles. IEEE Trans. Intell. Transport. Syst. 19, 92-101. doi: 10.1109/TITS.2017.2697044

Hatzopoulou, M., and Miller, E. J. (2010). Linking an activity-based travel demand model with traffic emission and dispersion models: transports contribution to air pollution in Toronto. Transport. Res. D Transport Environ. 15, 315-325. doi: 10.1016/j.trd.2010.03.007

Herrmann, A., Liu, M., Pilla, F. and Shorten, R. (2018). A new take on protecting cyclists in smart cities. IEEE Trans. Intell. Transport. Syst. 19, 3992-3999. doi: 10.1109/TITS.2018.2794298

Katsikouli, P., Ferraro, P., Timoney, D., and Shorten, R. (2019). On DICE-free smart cities, particulate matter, and feedback-enabled access control. arXiv [Preprint] arXiv:1906.10050.

Keuken, M., Hugo D. V. d. G., and Karin V. d. V. (2010). Non-exhaust emissions of PM and the efficiency of emission reduction by road sweeping and washing in the Netherlands. Sci. Tot. Environ. 408, 4591-4599. doi: 10.1016/j.scitotenv.2010.06.052

Kole, P. J., Lohr, A. J., Van Belleghem, F., and Ragas, A. (2017). Wear and tear of tyres: a stealthy source of microplastics in the environment. Int. J. Environ. Res. Public Health 14:1265. doi: 10.3390/ijerph14101265

Laden, F., Schwartz, J., Speizer, F. E., and Dockery, D. W. (2006). Reduction in fine particulate air pollution and mortality: extended follow-up of the Harvard Six Cities study. Am. J. Respirat. Crit. Care Med. 173, 667-672. doi: 10.1164/rccm.200503-443OC

Longo, M., Wahiba Y., and Dario Z. (2016). Team play between renewable energy sources and vehicle fleet to decrease air pollution. Sustainability 8:27. doi: 10.3390/su8010027

Mahmod, M., Van Arem, B., Pueboobpaphan, R., and De Lange, R. (2013). Reducing local traffic emissions at urban intersection using ITS countermeasures. IET Intell. Transport Syst. 7, 78-86. doi: 10.1049/ietits.2011.0222

Miranda, J., Makitalo, N., Garcia-Alonso, J, Berrocal, J., Mikkonen, T., Canal, C., et al. (2015). From the internet of things to the internet of people. IEEE Internet Comput. 19, 40-47. doi: 10.1109/MIC.2015.24

Nakamoto, S. (2008). Bitcoin: A Peer-to-Peer Electronic Cash System. Available online at: https://bitcoin.org/bitcoin.pdf

Overko, R., Ordonez-Hurtado, R. H., Zhuk, S., Ferraro, P., Cullen, A., and Shorten, R. (2019). Spatial positioning token (SPToken) for smart mobility. arXiv [Preprint] arXiv:1905.07681. doi: 10.1109/ICCVE45908.2019. 8964853

Panis, L. I., Broekx, S., and Liu, R. (2006). Modelling instantaneous traffic emission and the influence of traffic speed limits. Sci. Tot. Environ. 371, 270-285. doi: 10.1016/j.scitotenv.2006.08.017

Puthal, D., Malik, N., Mohanty, S. P., Kougianos, E., and Das, G. (2018). Everything you wanted to know about the blockchain: its promise, components, processes, and problems. IEEE Consumer Electron. Mag. 7, 6-14. doi: 10.1109/MCE.2018.2816299

Rai, A. C., Kumar, P., Pilla, F., Skouloudis, A. N., Di Sabatino, S., Ratti, C., et al. (2017). End-user perspective of low-cost sensors for outdoor air pollution monitoring. Sci. Tot. Environ. 607, 691-705. doi: 10.1016/j.scitotenv.2017.06.266

Salvat, O., Marez, P., and Belot, G. (2000). Passenger car serial application of a particulate filter system on a common rail direct injection diesel engine. SAE Trans. 109, 227-239. Available online at: http://www.jstor.org/stable/44745839

Schraufnagel, D. E., Balmes, J. D., Cowl, C. T., De Matteis, S., Jung, S. H., Mortimer, K., et al. (2019). Air pollution and noncommunicable disease. Chest 155, 409-441. doi: 10.1016/j.chest.2018.10.042

Suzuki, Y. (2011). A new truck-routing approach for reducing fuel consumption and pollutants emission. Transport. Res. D Trans. Environ. 16, 73-77. doi: $10.1016 /$ j.trd.2010.08.003

Sweeney, S., Ordonez-Hurtado, R., Pilla, F., Russo, G., Timoney, D. and Shorten, R. (2018). A context-aware e-Bike system to reduce pollution inhalation while cycling. IEEE Trans. Intell. Transport. Syst. 20, 704-715. doi: 10.1109/TITS.2018.2825436 
Terrill, E. R., Centea, M., Evans, L. R., and MacIsaac Jr, J. D. (2010). Dynamic Mechanical Properties of Passenger and Light Truck Tire Treads. Report No. DOT HS, 811:28.

Timmers, V. R., and Achten, P. A. (2016). Non-exhaust PM emissions from electric vehicles. Atmos. Environ. 134, 10-17. doi: 10.1016/j.atmosenv.201 6.03.017

Valavanidis, A., Fiotakis, K., and Vlachogianni, T. (2008). Airborne particulate matter and human health: toxicological assessment and importance of size and composition of particles for oxidative damage and carcinogenic mechanisms. J. Environ. Sci. Health C 26, 339-362. doi: 10.1080/105905008024 94538

Visvizi, A., and Lytras, M. (2019). Smart Cities: Issues and Challenges: Mapping Political, Social and Economic Risks and Threats. Elsivier.

WHO (2013). Health Effects of Particulate Matter. Policy Implications for Countries in Eastern Europe, Caucasus and Central Asia.

Yu, B., Ma, Y., Xue, M., Tang, B., Wang, B., Yan, J., et al. (2017). Environmental benefits from ridesharing: a case of Beijing. Appl. Energy 191, 141-152. doi: 10.1016/j.apenergy.2017.01.052
Zambonelli, F. (2012). Toward sociotechnical urban superorganisms. Computer 45, 76-78. doi: 10.1109/MC.2012.280

Zheng, Z., Xie, S., Dai, H., Chen, X., and Wang, H. (2017). "An overview of Blockchain technology: architecture, consensus, and future trends," in IEEE International Congress on Big Data, 557-564.

Conflict of Interest: The authors declare that the research was conducted in the absence of any commercial or financial relationships that could be construed as a potential conflict of interest.

Copyright (c) 2020 Katsikouli, Ferraro, Richardson, Cheng, Anderson, Mallya, Timoney, Masen and Shorten. This is an open-access article distributed under the terms of the Creative Commons Attribution License (CC BY). The use, distribution or reproduction in other forums is permitted, provided the original author(s) and the copyright owner(s) are credited and that the original publication in this journal is cited, in accordance with accepted academic practice. No use, distribution or reproduction is permitted which does not comply with these terms. 